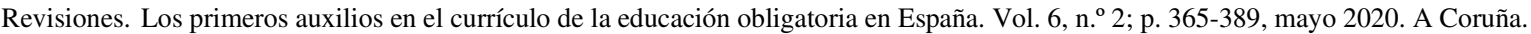
España ISSN 2386-8333

\title{
Los primeros auxilios en el currículo de la educación obligatoria en España
}

\section{First aid in the curriculum of compulsory education in Spain}

\author{
Lois Rodríguez-Lorenzo ${ }^{1}$; Brais Ruibal-Lista ${ }^{2}$; Sofía Toro ${ }^{2}$
}

${ }^{1}$ Grupo de Aprendizaje y control del movimiento humano, Universidade da Coruña ${ }^{2}$ Grupo de Investigación en Actividades Acuáticas y Socorrismo,Universidade da Coruña

${ }^{3}$ Facultad de Ciencias de la Actividad Física y del Deporte, Universidad Católica San Antonio de Murcia

Autor de correspondencia: Lois Rodríguez-Lorenzo

Contacto: lois.rodriguez@edu.xunta.gal

Cronograma editorial: Artículo recibido: 05/12/2019 Aceptado: 07/04/2020 Publicado: 01/05/2020 DOI: https://doi.org/10.17979/sportis.2020.6.2.5831 


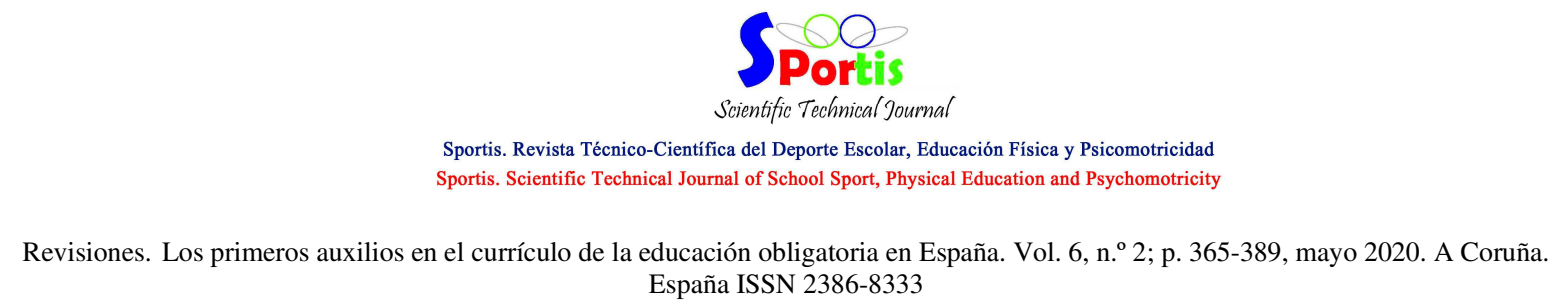

\title{
Resumen
}

La formación en primeros auxilios es fundamental para poder intervenir en un primer momento en situaciones de emergencia y es por ello que numerosas instituciones han señalado a la educación obligatoria como una de las claves para llevar a cabo dicha formación. En esta investigación se ha analizado la evolución de la presencia de los primeros auxilios en la legislación educativa en España, que han ido perdiendo peso en el currículo educativo desde la LGE, en 1970, hasta la LOE, en 2006, especialmente en el bachillerato y han recuperado su importancia desde ese año hasta la actual LOMCE, de 2013, donde se incluyen estos contenidos en la educación primaria y la educación secundaria obligatoria. Aunque los contenidos se incluyen principalmente en la asignatura de educación física, actualmente se dividen en varias asignaturas del currículo. La evidencia científica actual nos dice que, aunque estos contenidos están presentes en el currículo, no se suelen aplicar por falta de material y también en la formación en el profesorado para impartirlos, sin embargo, ciertas comunidades autónomas aportan material y formación para que puedan ser los propios docentes los que impartan las maniobras de primeros auxilios en las escuelas. Por todo esto, creemos que los primeros auxilios deben ser un contenido obligatorio y relevante en las escuelas y que deben ser los docentes, formados previamente, los que impartan dichos contenidos.

\section{Palabras clave}

Accidentes; currículo; educación; legislación; primeros auxilios.

\begin{abstract}
Learning first aid is essential to be able to act in an emergency situation and that is why many institutions have pointed at compulsory education as one of the keys to carry out such learning. The evolution of the presence of first aid in educational legislation in Spain has been analyzed in this research. These contents have been losing weight in the educational curriculum from the LGE, in 1970 to the LOE, in 2006, especially in the baccalaureate and have increased its importance since that year until the current LOMCE, 2013, which includes these contents in primary education and compulsory secondary education. Although the contents are mainly included in the subject of physical education, they are currently divided into several subjects of the curriculum. The current scientific evidence tells us that, although these contents are present in the curriculum, they are not usually taught due to the lack of material and training for teachers to teach them. However, certain autonomous communities provide material and training, so that the teachers can teach by themselves the first aid techniques in schools. For all this, we believe that first aid must be a compulsory and relevant content in schools, and teachers, trained in advance, must teach these contents.
\end{abstract}

Para citar este artículo utilice la siguiente referencia: Rodríguez-Lorenzo, L.; Ruibal-Lista, B.; Toro, S. (2020). Los primeros auxilios en el currículo de la educación obligatoria en España. Sportis Sci J, 6 (2), 365-389. DOI: https://doi.org/10.17979/sportis.2020.6.2.5831 


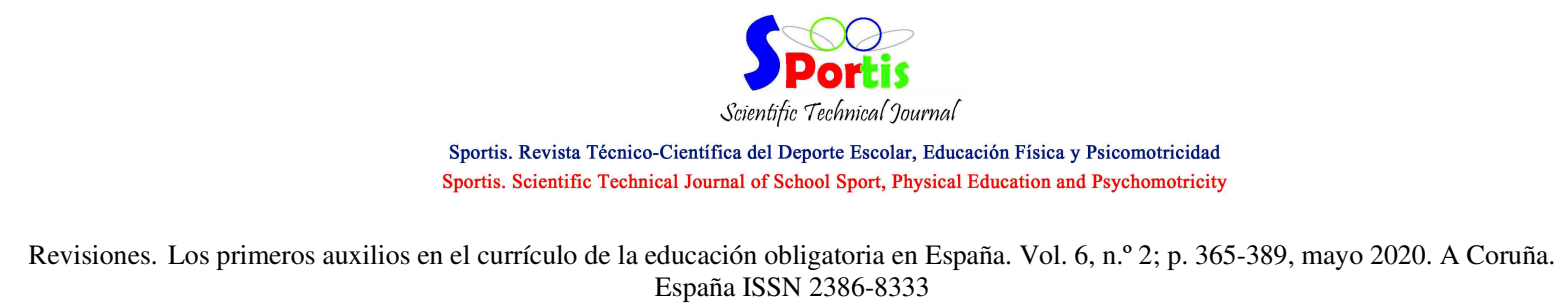

\section{Key Words}

Accidents; curriculum; education; first aids; legislation.

\section{Introducción}

Los primeros auxilios son todas las acciones que se realizan para ayudar una persona, que ha sufrido un accidente o una enfermedad repentina, antes de que reciba atención médica especializada (Wilks \& Pendergast, 2017). Se proporcionan en el lugar del accidente, de forma instantánea o lo más rápido posible, utilizando solamente los medios que se tengan alrededor (Tenorio, 2007). Su aplicación puede reducir la gravedad de las lesiones y salvar vidas (IFRC, 2009).

De acuerdo con la Organización Mundial de la Salud, "un accidente es un acontecimiento, independientemente de la voluntad humana, (...) que pueda alcanzar o no a una persona, y que origina que la persona tenga que solicitar posteriormente asistencia médica, independientemente del daño corporal o mental" (DADO, 2011).

En los centros escolares se producen un número elevado de accidentes relacionados con la práctica de actividad física y deportiva (organizada y no organizada), siendo en la mayoría de los casos de carácter leve (Abernethy \& MacAuley, 2003; Linakis, 2006). Los accidentes más habituales que se suelen producir en los centros escolares son, entre otros: choques, caídas, heridas, hemorragias, cortes, atragantamientos, asfixias, quemaduras, daños musculares o pinchazos, (Muñoz, 2009). En las situaciones que requieren una intervención de urgencia por accidente, los docentes deberán de conocer los protocolos sobre cómo actuar (Conducta PAS: Proteger-Avisar-Socorrer), y si es necesario, estar preparados para proporcionar primeros auxilios al alumno.

Fuera del entorno escolar, es conocida la morbilidad y la mortalidad por accidentes de tráfico y/o laborales (Peiró-Pérez, 2006), gracias a que existe una gran difusión en los principales medios de comunicación. En el año 2017 se registraron en España 102.233 accidentes de tráfico con víctimas, que provocaron 129.616 heridos no hospitalizadas, 9.546 heridos hospitalizados y 1.830 fallecidos (DGT, 2017). En ese mismo año se contabilizaron 596.606 accidentes laborales con baja en jornada de trabajo, de los cuales 515.082 fueron de carácter leve, 3.904 de carácter grave y 496 fueron causa directa de la muerte del trabajador (MESS, 2017). Sin embargo, la repercusión mediática de los accidentes del en el ámbito

Para citar este artículo utilice la siguiente referencia: Rodríguez-Lorenzo, L.; Ruibal-Lista, B.; Toro, S. (2020). Los primeros auxilios en el currículo de la educación obligatoria en España. Sportis Sci J, 6 (2), 365-389. DOI: https://doi.org/10.17979/sportis.2020.6.2.5831 


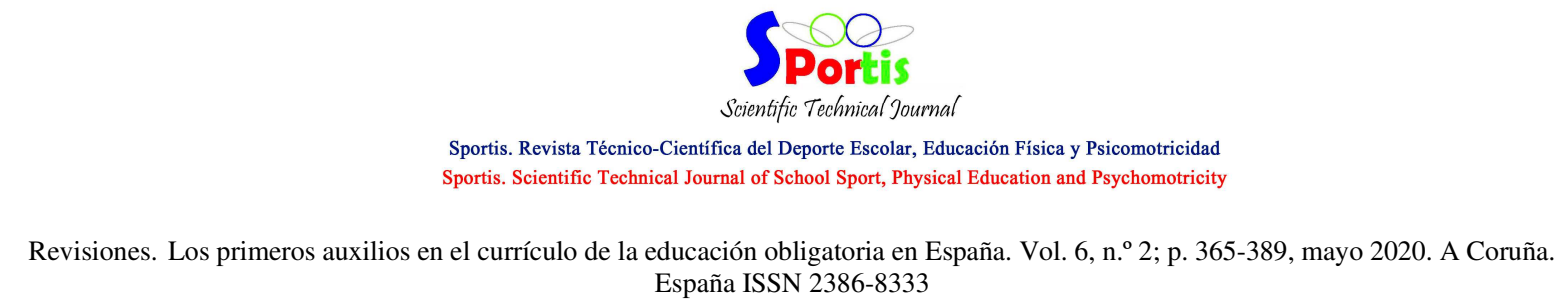

doméstico y en las actividades de ocio es muy inferior, a pesar de que suponen la cuarta causa de mortalidad en la Unión Europea (DADO, 2011). En España, según un estudio realizado por el Instituto Nacional de Consumo, se estima que 2.576.601 ciudadanos sufrieron un accidente doméstico o de ocio durante el año 2011 (DADO, 2011), de los cuales el 87,5\% recibió algún tipo de tratamiento como consecuencia del siniestro.

Cualquier persona puede presenciar o verse involucrada en un accidente doméstico, laboral o de tráfico. Nuestro deber como ciudadano será prestar ayuda tal y como se recoge en el artículo 194 del Código Penal: "El que no socorriere a una persona que se halle desamparada y en peligro manifiesto y grave, cuando pudiere hacerlo sin riesgo propio ni de terceros, será castigado con la pena de multa de tres a doce meses". Por lo tanto, la formación en primeros auxilios es fundamental para todos y cada uno de los ciudadanos. Sin embargo, según la Sociedad Española de Medicina de Urgencia y Emergencias, los españoles son los ciudadanos peor preparados de Europa para hacer frente a una situación que requiera proporcionar primeros auxilios. Además, un tercio la población española reconoce no sentirse para nada seguro en caso de tener que hacerlo (SEMES, 2017).

La educación y la formación, especialmente de los más jóvenes, es fundamental para la prevención de accidentes (Ruíz-Fernández, 2016). Desde la administración educativa se debe fomentar este tipo de enseñanzas, debido a que generalmente los progenitores no cuentan con una formación adecuada para transmitirlas (Sánchez, Rodríguez \& Sánchez, 2008).

El hecho de que prácticamente la totalidad de la población tiene que pasar por el sistema educativo en algún momento de su vida, hace que la educación obligatoria sea un lugar idóneo para formar al mayor número de ciudadanos posible en materia de primeros auxilios. Por lo tanto, en el ámbito escolar los primeros auxilios deberán formar parte del bagaje de conocimientos científicos teórico-prácticos que deberán conocer y manejar los docentes, y deberán estar recogidos en el currículum como parte de los aprendizajes que deberán adquirir los alumnos en la escuela.

El objetivo del presente trabajo será analizar la presencia e importancia de los primeros auxilios en el currículo educativo español y su evolución a lo largo de las diferentes leyes de educación.

Para citar este artículo utilice la siguiente referencia: Rodríguez-Lorenzo, L.; Ruibal-Lista, B.; Toro, S. (2020). Los primeros auxilios en el currículo de la educación obligatoria en España. Sportis Sci J, 6 (2), 365-389. DOI: https://doi.org/10.17979/sportis.2020.6.2.5831 


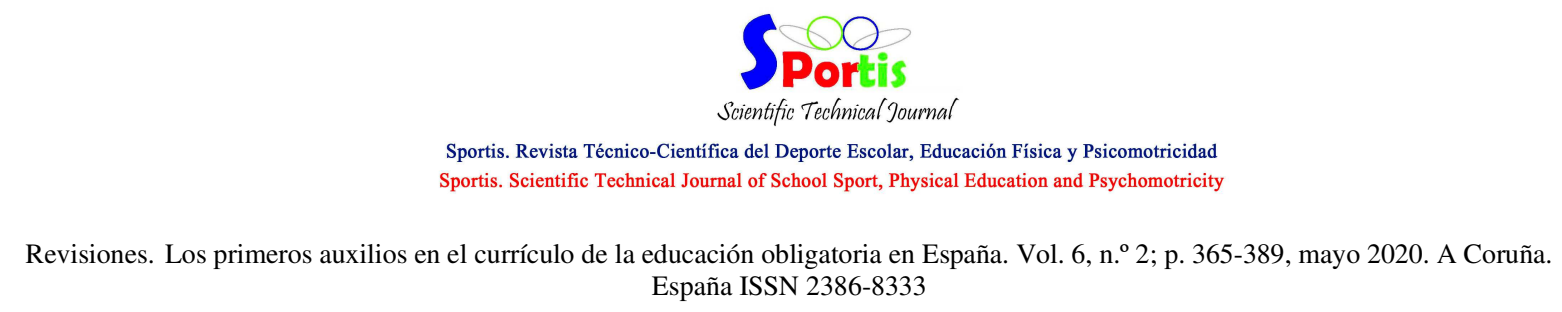

\section{Material y método}

Se ha realizado una revisión sistemática de la legislación educativa para identificar la presencia de los primeros auxilios en el currículo. El análisis curricular realizado se enmarca dentro del primer nivel de concreción curricular, que es establecido por las administraciones educativa del estado a través de la legislación vigente.

En una primera fase se ha analizado las disposiciones de carácter general de ámbito estatal de la educación obligatoria (primaria y secundaria) y el bachillerato, que afectan a los elementos que configuran el currículo educativo, desde 1960 hasta 2020. La revisión fue realizada por un experto en legislación educativa utilizando la plataforma proporcionada por la Agencia Estatal Boletín Oficial del Estado. Esta plataforma cuenta con el Identificador Europeo de Legislación-ELI, y permite acceder online a la normativa, publicada en los diarios y las bases de datos oficiales, en un formato normalizado. Se consultaron un total de 41 documentos legislativos, de los cuales 24 fueron seleccionados para su posterior análisis (5 leyes, 12 Reales Decretos, 2 Decretos y 5 Órdenes).

En una segunda fase, tres expertos realizaron una exhaustiva lectura cada uno de estos 24 documentos buscando aspectos relacionados con los primeros auxilios. Posteriormente cada uno de ellos realizó un último filtro mediante una búsqueda informática utilizando las siguientes palabras: "primeros auxilios", "socorrismo", "lesiones", “accidentes", "prevención”, “actuación”, “ayuda”, “emergencia”. A continuación, se realizó una puesta en común elaborado 5 tablas (tablas 1-5), que muestran la presencia de los primeros auxilios en los elementos curriculares en cada uno de los documentos analizados. Debido a que los constantes cambios que ha sufrido la legislación educativa en España, han afectado a los elementos que configuran el currículo educativo, consideramos necesario explicar y describir cómo ha ido evolucionando el currículo y el Sistema Educativo a través de normativa vigente en cada momento.

Finalmente, para analizar la evolución y la importancia de los primeros auxilios en el currículo educativo se realizó una búsqueda de estudios científicos relacionados con los primeros auxilios en las siguientes bases de datos: Web of Science, Google Schollar, Scopus y Dialnet. Para su realización se utilizaron las siguientes palabras clave: "primeros auxilios", 
"socorrismo", "escuela", "RCP”. Se consultaron un total de 82 documentos de los cuales 37 fueron incluidos en esta revisión.
\end{abstract}

\title{
3. Resultados \\ Antecedentes históricos
}

La Ley 14/1970, de 4 de agosto, General de Educación y Financiamiento de la Reforma Educativa (LGE), vino a significar un intento de ordenar una escuela tradicional basada en concepciones autoritarias mediante sistema educativo centralizado basado en la tradición educativa liberal, que trajo consigo una uniformidad en la enseñanza. Esta Ley estructura todo el sistema educativo español en cuatro niveles: Preescolar, Educación General Básica, Enseñanzas Medias (Bachillerato y Formación profesional) y Enseñanza Universitaria.

Con el desarrollo de la Ley General de Educación de 1970 apareció la Orden de 22 de marzo de 1975 que estable las bases de programación de las diversas materias del Bachillerato en el denominado "plan de estudios", que determina de forma conjunta los contenidos, objetivos y orientaciones metodológicas de cada materia y curso académico. El programa de cada asignatura era un programa cerrado y existía una confusión ya que se mezclaban objetivos y contenidos. Dentro del programa de la materia de Educación Física se encuentran las primeras referencias los primeros auxilios para cada uno de los 3 cursos del Bachillerato, bajo la denominación de "socorrismo deportivo" (Tabla 1). El socorrismo puede definirse como la organización y adiestramiento para prestar socorro en caso de accidente (RAE, 2014). En esa época, el socorrismo deportivo exigía un dominio de conocimientos en materia de primeros auxilios y técnicas de salvamento (Abraldes, 2007), y su enfoque pedagógico en la legislación educativa tenía un marcado carácter práctico y competitivo. Sin embargo, los contenidos de "socorrismo" desaparecen del plan de estudios de bachillerato con la actualización del programa de la materia de Educación Física y Deportiva en la Orden de 18 de septiembre de 1987, y la legislación educativa no volverá a hacer referencia directa al socorrismo deportivo.

Durante los años 70 se produce un vacío normativo en la Educación General Básica (EGB), ya que no se marcan objetivos y contenidos oficiales hasta que se produjo la reforma 


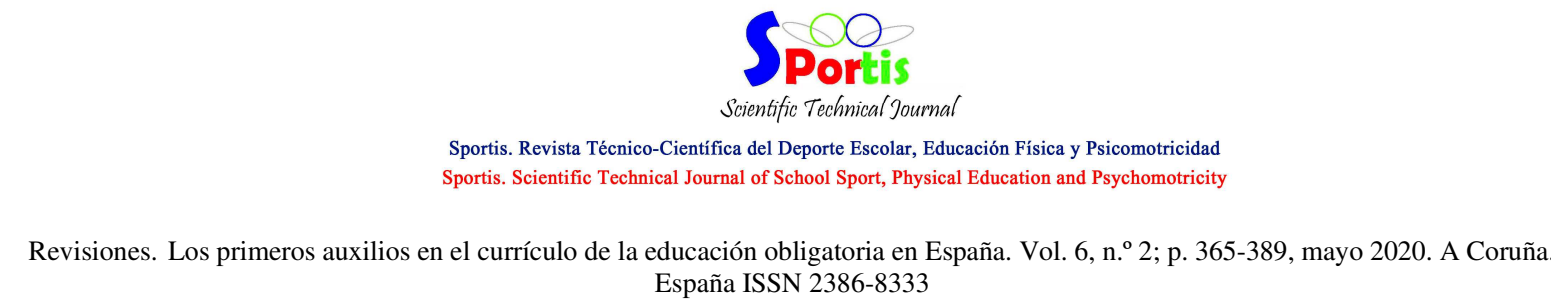

curricular parcial de 1980-1982. A partir de los 80 se comienza a introducir la programación por objetivos, en los RRDD de enseñanzas mínimas se establecen los objetivos que todos los alumnos del territorio español han de conseguir al terminar cada ciclo de la EGB, y que se conocen con el nombre de "Objetivos terminales". En el Real Decreto 69/1981, se ordenó la Educación General Básica (EGB) en tres ciclos (inicial: $1^{\circ}$ y $2^{\circ}$ de EGB, medio: $3^{\circ}, 4^{\circ}$ y $5^{\circ}$ de EGB y superior: $6^{\circ}, 7^{\circ}$ y $8^{\circ}$ de EGB) y se fijaron las enseñanzas mínimas para el Ciclo Inicial, no haciendo ninguna referencia a los primeros auxilios. Aproximadamente un año después se fijaron, en Real Decreto 710/1982, las enseñanzas mínimas para el ciclo medio de la EGB, y aparecen las primeras referencias a cómo actuar, de forma teórica, en caso de diferentes accidentes dentro de la materia "Ciencias de la naturaleza" todavía bajo la denominación de "medidas de socorro" (Tabla 1). El término "socorro" puede definir como la "Acción y efecto de socorrer" que a su vez significa "Ayudar, favorecer en un peligro o necesidad" (RAE,2014). En el Real Decreto 3087/1982, se establecieron las enseñanzas mínimas para el ciclo superior, se utiliza por primera vez la denominación de "primeros auxilios" y estos adquieren una gran importancia teórico-práctica dentro Bloque Temático de "Conocimiento de sí mismo" y "técnicas de trabajo" de la materia de "Ciencias de la naturaleza y tecnología" (Tabla 1).

La Orden de 17 de enero de 1981 (Ciclo inicial), la Orden de 6 de mayo de 1982, (Ciclo Medio) y la Orden de 25 de noviembre de 1982 (Ciclo Superior), complementan y desarrollan las los Decretos de enseñanzas mínimas, incorporando los programas de las materias de Educación Física y de Educación Artística, y distribuyendo los objetivos terminales por cursos. En la documentación correspondiente a los dos primeros ciclos de la EGB, no aparecen ninguna referencia a los primeros auxilios. Sin embargo, en la Orden de 25 de noviembre de 1982 aparecen 6 menciones enfocadas conocer y practicar diferentes medidas de primeros auxilios, dentro de la materia de "Ciencias de la naturaleza y tecnología" en los 3 cursos del Ciclo Superior de la EGB (Tabla 1). 


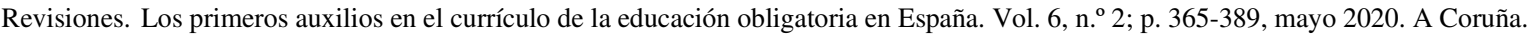
España ISSN 2386-8333

Tabla 1. Elementos curriculares relacionados con los primeros auxilios en la normativa legislativa de la Ley General de Educación (LGE)

\begin{tabular}{|c|c|c|}
\hline \multicolumn{3}{|c|}{ Real Decreto 710/1982 } \\
\hline $\begin{array}{l}\text { Ciclo medio } \\
\text { Educación } \\
\text { General } \\
\text { Básica }\end{array}$ & $\begin{array}{l}\text { CIENCIAS DE LA NATURALEZA: } \\
\text { Bloque temático 1: Conocimiento de sí } \mathrm{n} \\
\text { 1.2. (...) "Conocer las medidas de socor } \\
\text { 1.7. (...) "Conocer las medidas eleme } \\
\text { heridas y quemaduras". }\end{array}$ & $\begin{array}{l}\text { smo } \\
\text { en caso de fracturas y desgarramientos". } \\
\text { ales de socorro, en caso de insolación, hemorragia, }\end{array}$ \\
\hline $\begin{array}{c}\text { Ciclo superior } \\
\text { Educación } \\
\text { General } \\
\text { Básica }\end{array}$ & $\begin{array}{l}\text { Real Decreto 3087/1982 } \\
\text { CIENCIAS DE LA NATURALEZA Y } \\
\text { TECNOLOGIA: } \\
\text { Bloque temático 1: Conocimiento de sí } \\
\text { mismo } \\
\text { 1.1. (...) "Conocer y practicar algunas } \\
\text { medidas de higiene y primeros auxilios } \\
\text { del aparato digestivo". } \\
\text { 1.2. (...) "Conocer y practicar medidas } \\
\text { de higiene y de primeros auxilios de los } \\
\text { órganos excretores (pulmón, riñón, } \\
\text { piel), así como de los aparatos } \\
\text { circulatorio y respiratorio". } \\
\text { 1.3. (...) "Conocer y practicar algunas } \\
\text { medidas de higiene y de primeros } \\
\text { auxilios de los órganos de los sentidos } \\
\text { y del sistema nervioso". } \\
\text { Bloque temático } 4: \text { Técnicas de trabajo } \\
\text { 4.5. (...) "Conocer los primeros } \\
\text { auxilios en caso de cortes, quemaduras, } \\
\text { intoxicaciones, fracturas..." }\end{array}$ & 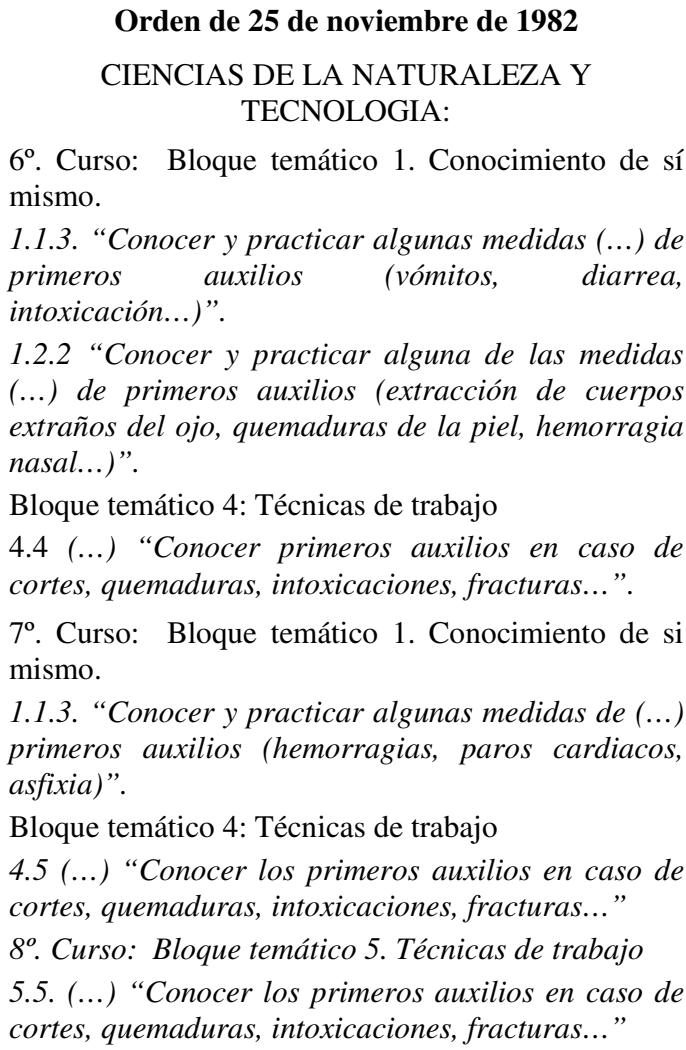 \\
\hline
\end{tabular}

\section{Orden de 22 de marzo de 1975}

EDUCACIÓN FÍSICA Y DEPORTIVA

Bachillerato $1^{\circ}$ Curso: "Práctica de socorrismo deportivo y práctica de aire libre".

$2^{\circ}$ Curso: "Práctica de socorrismo deportivo".

$3^{\circ}$ Curso: "Práctica de competición de socorrismo deportivo y general".

Extraído de: RD 710/1982; RD 3087/1982; Orden 25 de noviembre de 1982; Orden de 22 de marzo de 1975.

En 1990 se promulga la Ley de Ordenación General del Sistema Educativo (LOGSE) constituyendo una apuesta por colocar a España a la altura de los países europeos en materia educativa. La LOGSE organiza las enseñanzas de régimen general en las siguientes etapas: Educación Infantil, Educación primaria, Educación Secundaria, formada por la Educación 


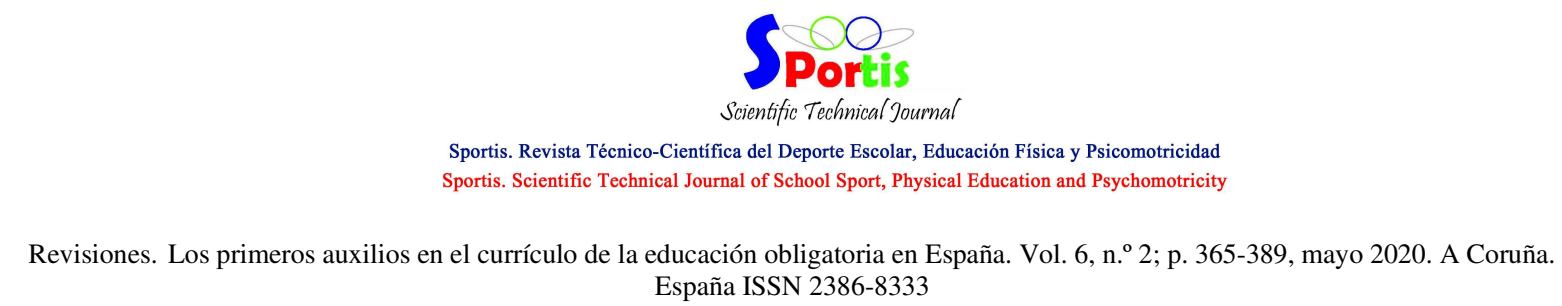

Secundaria Obligatoria (ESO), el Bachillerato, la Formación Profesional (FP), y Educación Universitaria.

La entregada en vigor de la LOGSE supuso un cambio importante en el panorama educativo nacional. Se pasó de un plan de estudios cerrado y centralizado a un currículo abierto y descentralizado, con diferentes niveles de concreción. En su artículo 4.1 define currículo como "el conjunto de objetivos, contenidos, métodos pedagógicos y criterios de evaluación de cada uno de los niveles, etapas, ciclos, grados y modalidades del sistema educativo que regulan la práctica docente". El Gobierno establecía entre el 55-65\% de los aspectos básicos del currículo de cada una de las etapas del sistema educativo, que constituirán las enseñanzas mínimas, de carácter preceptivo para todas las Comunidades Autónomas (CCAA). De esta forma, las CCAA, disponían de una margen de decisión para adaptar currículo de cada etapa del sistema educativo a su ámbito territorial.

En el presente trabajo nos centramos en el análisis de los Decretos de enseñanzas mínimas de ámbito estatal, que establecen los objetivos, los contenidos y los criterios de evaluación correspondientes de cada materia y nivel educativos. En el Real Decreto 1006/1991, de educación primaria, y en el Real Decreto 1178/1992, de bachillerato, no aparecen ninguna referencia a los primeros auxilios. Únicamente, en Real Decreto 1345/1991, de educación secundaria, aparece una breve referencia a cómo actuar en caso de accidente dentro de la materia de Educación Física (Tabla 2), que posteriormente desaparece en la actualización curricular que se establece en el Real Decreto 3473/2000.

Tabla 2

Elementos curriculares relacionados con los primeros auxilios en la normativa legislativa de la Ley de Ordenación General del Sistema Educativo (LOGSE)

\begin{tabular}{ll}
\hline & \multicolumn{1}{c}{ Real Decreto 1345/1991 } \\
& EDUCACIÓN FÍSICA \\
Educación & Contenidos procedimentales \\
Secundaria & Bloque 1: Condición física. \\
Obligatoria & 5. "Prevención y actuación en caso de accidentes en actividades físicas”. \\
\hline Extraído de: RD 1345/1991.
\end{tabular}

Por lo tanto, podemos afirmar que con la entrada en vigor de la LOGSE los primeros auxilios perdieron importancia en el currículo educativo. Desapareciendo totalmente de la educación primaria y de las materias de ciencias, conservándose con poco peso en la 


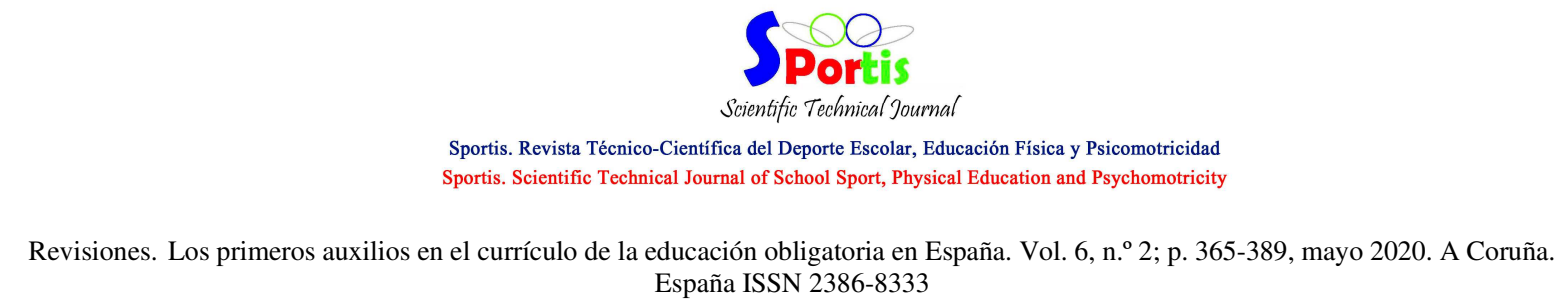

educación secundaria dentro de la materia de educación física, hasta su completa desaparición en el 2000.

En 2002 se promulga la Ley Orgánica de Calidad de la Educación (LOCE), pero debido a la gran oposición política y social esta ley nunca llegó a aplicarse, por lo que no la incluiremos en nuestro análisis.

En 2006 se aprueba la Ley Orgánica de Educación (LOE), que nace para mejorar la calidad y la eficacia del sistema educativo, adaptarse los nuevos cambios sociales y a la nueva normativa europea. La estructura del sistema educativo no sufre grandes cambios. El cambio más significativo es la incorporación de las competencias básicas como un nuevo componente del currículo educativo, que permiten desde un planteamiento integrador, orientar y poner acento en aquellos aprendizajes que se consideran imprescindibles. Las competencias básicas se desarrollan en la etapa Primaria para ser alcanzadas en la de ESO, donde tendrán una especial imporancia. Todas las materias deben contribuir al desarrollo de las competencias básicas, a fin de que al terminar la enseñanza obligatoria sean adquiridas por todo el alumnado para "poder lograr su realización personal, ejercer la ciudadanía activa, incorporarse a la vida adulta y ser capaz de desarrollar un aprendizaje permanente a lo largo de la vida".

La LOE define en su artículo 6 en currículo como "la regulación de los elementos que determinan los procesos de enseñanza y aprendizaje para cada una de las enseñanzas", es decir "el conjunto de objetivos, competencias básicas, contenidos, métodos pedagógicos y criterios de evaluación de cada etapa". Para asegurar una formación común y garantizar la homologación de los títulos, en Gobierno fija unas enseñanzas mínimas (un 55-65\% de los elementos del currículo). La presencia de los primeros auxilios en el currículo educativo de la LOE, desarrollado en los Reales Decretos de enseñanzas mínimas de Educación Primaria y de la ESO, ha aumentado considerablemente (Tabla 3), recuperando importancia en las materias de ciencias naturales y de educación física en el último ciclo de cada etapa educativa. En las materias de ciencias naturales se hace una breve mención general a los primeros auxilios dentro del Bloque de contenidos de la "Las personas y la Salud". En educación física se trata, de una forma más práctica y específica, como actuar ante las lesiones más comunes que se pueden producir en durante la práctica de actividad física o la vida cotidiana (limpiar una 


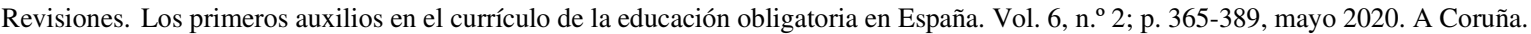
España ISSN 2386-8333

herida o aplicar frío ante un traumatismo músculo-esquelético). Además, los primeros auxilios aparecen por primera vez dentro de una materia optativa (Alimentación, nutrición y salud) con un enfoque teórico-prácticos, que aunque el currículo no especificaba, era propicio para enseñar cómo actuar frente a un atragantamiento mediante la maniobra de Heimlich. Sin embargo, en el Real Decreto 1467/2007, por el que se establece la estructura del bachillerato y se fijan sus enseñanzas mínimas, no aparecen ninguna referencia a los primeros auxilios.

Tabla 3. Elementos curriculares relacionados con los primeros auxilios en la normativa legislativa de la Ley Orgánica de Educación (LOE)

\section{Real Decreto 1513/2006}

Educación Primaria CONOCIMIENTO DEL MEDIO NATURAL, SOCIAL Y CULTURAL:

$3^{\circ}$ Ciclo

Bloque 3. La salud y el desarrollo personal

"Conocimiento de primeros auxilios para saber ayudarse y ayudar a los demás".

\begin{tabular}{|c|c|}
\hline & Real Decreto 1631/2006 \\
\hline & $\begin{array}{l}\text { CIENCIAS DE LA NATURALEZA } \\
3^{\circ} \text { Curso: Contenidos del Bloque 5. Las personas y la salud. } \\
\text { "Higiene y prevención de las enfermedades. Primeros auxilios. Valoración de la } \\
\text { importancia de los hábitos saludables" }\end{array}$ \\
\hline & $\begin{array}{l}\text { EDUCACIÓN FÍSICA } \\
4^{\circ} \text { Curso: Contenidos del Bloque 1. Condición física y salud. } \\
\text { "Primeras actuaciones ante las lesiones más comunes que pueden manifestarse en la } \\
\text { práctica deportiva". }\end{array}$ \\
\hline \multirow{3}{*}{$\begin{array}{l}\text { Educación } \\
\text { Secundaria }\end{array}$} & $\begin{array}{l}\text { Criterios de evaluación: } \\
\text { 4. "Resolver supuestos prácticos sobre las lesiones que se pueden producir en la vida } \\
\text { cotidiana, en la práctica de actividad física y en el deporte, aplicando unas primeras } \\
\text { atenciones". }\end{array}$ \\
\hline & $\begin{array}{l}\text { "El alumnado demostrará tener un conocimiento teórico-práctico básico de las } \\
\text { actuaciones que deben llevarse a cabo ante lesiones que puedan producirse en su } \\
\text { entorno habitual y, concretamente, en la práctica de actividadfísica". }\end{array}$ \\
\hline & $\begin{array}{l}\text { "Se incidirá muy especialmente en los aspectos preventivos y en aquéllos que evitan la } \\
\text { progresión de la lesión. El alumnado aprenderá por ejemplo a limpiar una herida o } \\
\text { aplicar frío ante un traumatismo músculo-esquelético". }\end{array}$ \\
\hline
\end{tabular}

ALIMENTACIÓN, NUTRICIÓN Y SALUD (optativa en $4^{\circ}$ Curso)

Contenidos del Bloque 1. Salud y enfermedad. Hábitos saludables

"Primeros auxilios. Conducta a seguir en casos de emergencia. Técnicas básicas de primeros auxilios.”

Criterios de evaluación

5. "Argumentar la importancia de los hábitos y estilos de vida saludables para poder conseguir un mejor grado de salud y aplicar técnicas de primeros auxilios en aquellas situaciones en que resulten necesarias".

(...) "Asimismo se valorará si el alumnado sabe aplicar las técnicas básicas de primeros auxilios que se requieran en una situación determinada".

Extraído de: RD 1513/2006; RD 1631/2006

Para citar este artículo utilice la siguiente referencia: Rodríguez-Lorenzo, L.; Ruibal-Lista, B.; Toro, S. (2020). Los primeros auxilios en el currículo de la educación obligatoria en España. Sportis Sci J, 6 (2), 365-389. DOI: https://doi.org/10.17979/sportis.2020.6.2.5831 


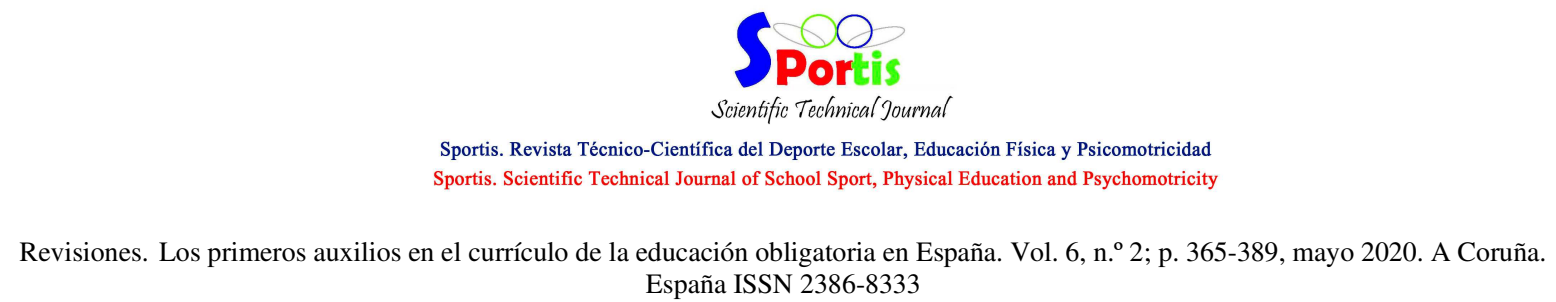
España ISSN 2386-8333

\section{Estado actual}

En 2013 se aprueba la Ley Orgánica para la Mejora de la Calidad Educativa (LOMCE), una ley controvertida que reforma la LOE y que se aprueba sin un amplio consenso comunidad educativa. Aunque la LOMCE supone una continuación del enfoque curricular por competencias, encontramos un cambio de orientación pedagógica importante: mientras la LOE ponía su foco de atención en una enseñanza por competencias orientada al aprendizaje del alumnado, la LOMCE pone su énfasis en una evaluación por competencias situada directa y predominantemente sobre el aprendizaje del alumnado (Molina, ValencianoValcárcel, \& Úbeda-Colomer, 2016).

La LOMCE reduce a siete las competencias y las define como capacidades, bajo la denominación de "competencias clave", (tabla 1) debiendo ser adquiridas al finalizar el Bachillerato.

El cambio curricular más significativo es la incorporación de las "estándares de aprendizaje evaluables" como un nuevo componente del currículo educativo, que en el Real Decreto 1105/2014, de 26 de diciembre, define como "especificaciones de los criterios de evaluación que permiten definir los resultados de aprendizaje, y que concretan lo que el estudiante debe saber, comprender y saber hacer en cada asignatura; deben ser observables, medibles y evaluables y permitir graduar el rendimiento o logro alcanzado".

Los reales decretos que regulan el currículo básico de la Educación Primaria (Real Decreto 126/2014), y de la ESO y del Bachillerato (Real Decreto 1105/2014) diferencian tres tipos de asignaturas: troncales, específicas y de libre configuración autonómica. En el bloque de asignaturas troncales se garantizan por etapa en (Educación primaria) y por ciclo (en ESO) unos contenidos comunes para todo el alumnado que se definen, para cada asignatura, en el currículo básico junto con los criterios de evaluación y los estándares de aprendizaje evaluables. En asignaturas específicas, como es el caso de la Educación Física, únicamente se establecen los criterios de evaluación y los estándares de aprendizaje evaluables, dejando la concreción de los contenidos y objetivos y la carga horaria a las Comunidades Autónomas y los centros educativos. (Flórez, Romero-Martín, \& Izco, 2015). No obstante, tal y como refleja el estudio de Molina, Valenciano-Valcárcel, \& Úbeda-Colomer, (2016) “los decretos

Para citar este artículo utilice la siguiente referencia: Rodríguez-Lorenzo, L.; Ruibal-Lista, B.; Toro, S. (2020). Los primeros auxilios en el currículo de la educación obligatoria en España. Sportis Sci J, 6 (2), 365-389. DOI: https://doi.org/10.17979/sportis.2020.6.2.5831 


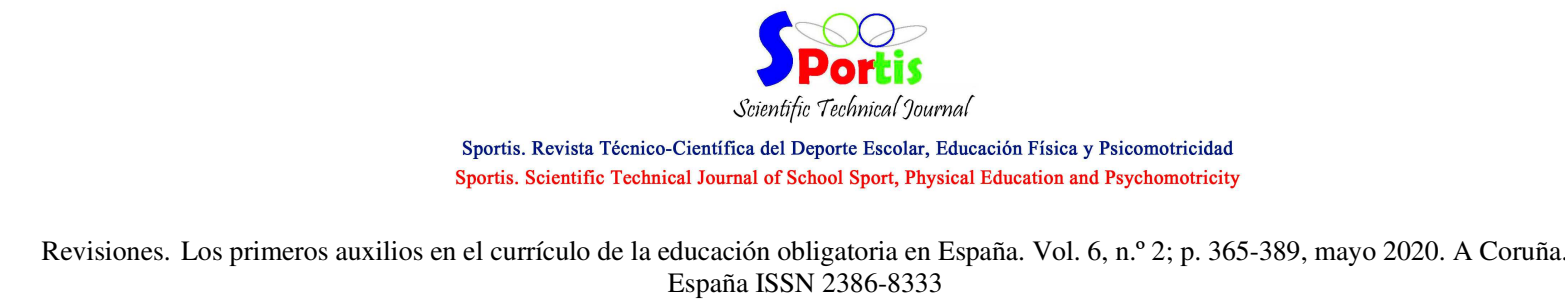

autonómicos de currículum (...) los han establecido para cada curso en relación con los criterios de evaluación y los estándares de aprendizaje que figuran en el $R D$ ”. En el presente trabajo tomaremos como ejemplo el Decreto 105/2014 (Educación Primaria), Decreto 86/2015 (ESO y Bachillerato) que concreta para la Comunidad Autónoma de Galicia los contenidos de las asignaturas específicas para en cada curso (Tabla 5), en relación a los estándares de aprendizaje y los criterios de evaluación de los Reales Decretos 126/2014 y 1105/2014 por los que se establece el currículo básico de la Educación Primaria y secundaria respectivamente (Tabla 4$)$.

Con la entrada en vigor de la LOMCE, los primeros auxilios ganan importancia dentro del currículo educativo de la educación primaria, estando presentes en tres asignaturas diferentes: Ciencias de la Naturaleza, Educación Física y Valores Sociales y Cívicos. Durante la etapa de educación secundaria, la legislación de ámbito estatal, corresponde a la materia de educación física impartir todos los contenidos relacionados con los primeros auxilios. En el caso de Galicia, los primeros auxilios también están presentes dentro del currículo de las materias de Valores Éticos y de Iniciación a la Actividad Emprendedora y Empresarial. Como venía ocurriendo en las anteriores leyes de educación, no aparecen ninguna referencia a los primeros auxilios en la etapa del Bachillerato. En el D86/20125 aparece, por primera vez, una mención específica a la conducta PAS (Proteger-Alertar-Socorrer), que es la secuencia principal de actuación ante un accidente: En primer lugar se debe proteger tanto el lugar, el accidentado y el propio interviniente. La alerta debe realizarse llamando al servicio de emergencia, por lo que se ha propuesto utilizar un solo número válido en toda Europa para facilitar el conocimiento del mismo en toda la población (Gómez-Sanabria et al, 2015). Por último, la acción de socorrer donde la valoración primaria y los conocimientos de primeros auxilios son determinantes. 


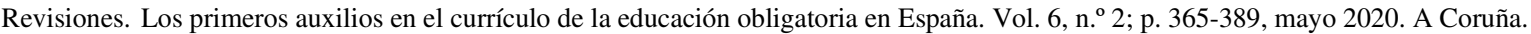
España ISSN 2386-8333

Tabla 4. Elementos curriculares relacionados con los primeros auxilios en la normativa legislativa de ámbito estatal de la Ley Orgánica para la Mejora de la Calidad Educativa (LOMCE)

Real Decreto 126/2014

CIENCIAS DE LA NATURALEZA

Bloque 2. El ser humano y la salud

Contenidos:

Conocimiento de actuaciones básicas de primeros auxilios.

Estándares de aprendizaje

3.7. Conoce y utiliza técnicas de primeros auxilios, en situaciones simuladas y reales.

\section{Educación}

Primaria

Estándares de aprendizaje

11.1. Explica y reconoce las lesiones y enfermedades deportivas más comunes, así como las acciones preventivas y los primeros auxilios.

\section{VALORES SOCIALES Y CÍVICOS}

Bloque 3. La convivencia y los valores sociales

Contenidos:

23. "Ser capaz de realizar primeros auxilios y tomar medidas preventivas valorando la importancia de prevenir accidentes domésticos".

Estándares de aprendizaje

23.3. "Conoce las prácticas de primeros auxilios".

\begin{tabular}{|c|c|}
\hline & Real Decreto 1105/2014 \\
\hline & $\begin{array}{c}\text { EDUCACIÓN FÍSICA } \\
1^{\circ} \text { Ciclo ESO }\left(1^{\circ}-3^{\circ} \text { Curso }\right)\end{array}$ \\
\hline & Estándares de aprendizaje \\
\hline Educación & $\begin{array}{l}\text { 9.2. "Describe los protocolos a seguir para activar los servicios de emergencia y de protección } \\
\text { del entorno". }\end{array}$ \\
\hline Secundaria & $4^{\circ} \mathrm{ESO}$ \\
\hline Obligatoria y & Criterios de evaluación \\
\hline Bachillerato & $\begin{array}{l}\text { 10. "Asumir la responsabilidad de la propia seguridad en la práctica de actividad física, } \\
\text { teniendo en cuenta los factores inherentes a la actividad y previendo las consecuencias que } \\
\text { pueden tener las actuaciones poco cuidadosas sobre la seguridad de los participantes". }\end{array}$ \\
\hline & Estándares de aprendizaje \\
\hline & 10.2. "Identifica las lesiones más frecuentes derivadas de la práctica de actividad física". \\
\hline & $\begin{array}{l}\text { 10.3. "Describe los protocolos que deben seguirse ante las lesiones, accidentes o situaciones de } \\
\text { emergencia más frecuentes producidas durante la práctica de actividades físico deportivas". }\end{array}$ \\
\hline
\end{tabular}

Extraído de: RD 126/2014; RD 1105/2014. 


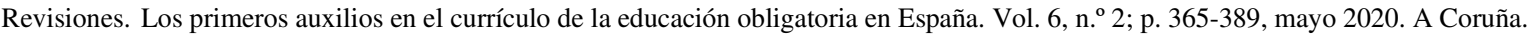
España ISSN 2386-8333

Tabla 5. Relación entre los elementos curriculares que tratan los primeros auxilios en Decreto 105/2014, por el que se establece el currículo básico de la Educación Primaria en Galicia

\begin{tabular}{|c|c|c|c|c|}
\hline Objetivo & Contenidos & $\begin{array}{l}\text { Criterios de } \\
\text { evaluación }\end{array}$ & $\begin{array}{l}\text { Estándares de } \\
\text { aprendizaje }\end{array}$ & Comp. \\
\hline \multicolumn{5}{|c|}{ EDUCACIÓN FÍSICA } \\
\hline \multicolumn{5}{|c|}{$5^{\circ}$ Curso: Bloque 5. Actividade física e saúde } \\
\hline $\mathrm{k}$ & $\begin{array}{l}\text { B5.5 } \\
\text { B5.6 }\end{array}$ & B5.3. & $\begin{array}{l}\text { 5.3.1. "Explica Las } \\
\text { lesiones y enfermedades } \\
\text { deportivas más comunes, } \\
\text { así como las acciones } \\
\text { preventivas y los primeros } \\
\text { auxilios básicos" }\end{array}$ & $\begin{array}{c}\text { CCL } \\
\text { CAA } \\
\text { CSC } \\
\text { CMCCT }\end{array}$ \\
\hline \multicolumn{5}{|c|}{ VALORES SOCIALES Y CÍVICOS } \\
\hline \multirow[b]{2}{*}{$\mathrm{k}$} & & $\begin{array}{l}\text { } 6^{\circ} \text { Curso } \\
\text { B3.15. "Ser capaz de } \\
\text { realizar primeros }\end{array}$ & & \multirow[b]{2}{*}{$\begin{array}{c}\text { CSC } \\
\text { CSIEE }\end{array}$} \\
\hline & $\begin{array}{l}\text { B3.23. } \\
\text { B3.24. } \\
\text { B3.25. Los primeros } \\
\text { auxilios }\end{array}$ & 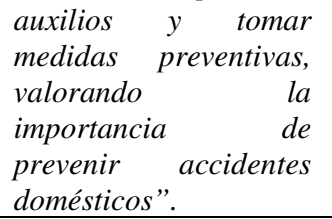 & $\begin{array}{ll}3.15 .2 . & \text { "Conoce las } \\
\text { prácticas de primeros } \\
\text { auxilios" }\end{array}$ & \\
\hline \multirow{2}{*}{\multicolumn{5}{|c|}{$\begin{array}{l}\text { CIENCIAS DE LA NATUREZA } \\
6^{\circ} \text { Bloque } 2 \text { - O ser humano e a saúde }\end{array}$}} \\
\hline & & & & \\
\hline $\begin{array}{c}\mathrm{a}, \mathrm{b}, \mathrm{c}, \mathrm{d}, \mathrm{h}, \\
\mathrm{k}, \mathrm{m}\end{array}$ & $\begin{array}{c}\text { B2.9.-B2.16 } \\
\text { B2.17. "Conocimiento y } \\
\text { actuaciones básicas de } \\
\text { primeros auxilios". }\end{array}$ & B2.3. & $\begin{array}{l}\text { 2.3.5. "Conoce las } \\
\text { técnicas de primeros } \\
\text { auxilios, en situaciones } \\
\text { simuladas y reales". }\end{array}$ & $\begin{array}{l}\text { CMCCT } \\
\text { CSC }\end{array}$ \\
\hline
\end{tabular}

\section{Comp: Competencias Clave.}

Extraído de: RD 105/2014.

Tabla 6. Relación entre los elementos curriculares que tratan los primeros auxilios en Decreto 86/201, por el que se establece el currículo básico de la Educación Secundaria en Galicia

\begin{tabular}{|c|c|c|c|c|}
\hline Objetivos & Contenidos & $\mathbf{C E}$ & Estándares de aprendizaje & Comp. \\
\hline \multicolumn{5}{|c|}{ EDUCACIÓN FÍSICA } \\
\hline \multicolumn{5}{|c|}{$1^{\circ} 2^{\circ}$ y $3^{\circ}$ Curso Bloque 1 . Contenidos comunes } \\
\hline $\mathrm{m}, \mathrm{n}, \mathrm{g}$ & $\begin{array}{l}\text { B1.11. "Protocolo básico } \\
\text { de actuación (PAS) en los } \\
\text { primeros auxilios". }\end{array}$ & & $\begin{array}{l}\text { 1.4.2. "Describe los protocolos para activar los } \\
\text { servicios de emergencia y de protección del } \\
\text { contorno". }\end{array}$ & CSIEE \\
\hline \multicolumn{5}{|c|}{$4^{\circ}$ Curso Bloque 1. Contenidos comunes } \\
\hline $\mathrm{a}, \mathrm{b}, \mathrm{g}, \mathrm{m}$ & $\begin{array}{l}\text { B1.5. "Primeras } \\
\text { actuaciones ante las } \\
\text { lesiones más comunes que } \\
\text { pueden manifestarse en la } \\
\text { práctica deportiva". } \\
\text { B1.6. "Protocolos básicos } \\
\text { de primeros auxilios". }\end{array}$ & B1.4. & $\begin{array}{l}\text { 1.4.2. "Identifica las lesiones más frecuentes } \\
\text { derivadas de la práctica de actividad física". } \\
\text { 1.4.3. "Describe los protocolos que deben } \\
\text { seguirse ante las lesiones, los accidentes o las } \\
\text { situaciones de emergencia más frecuentes } \\
\text { producidas durante la práctica de actividades } \\
\text { físico-deportivas". }\end{array}$ & CSIEE \\
\hline
\end{tabular}

INICIACIÓN A LA ACTIVIDAD EMPRENDEDORA Y EMPRESARIAL

$4^{\circ}$ Curso Bloque 1. Autonomía personal, liderazgo e innovación

Para citar este artículo utilice la siguiente referencia: Rodríguez-Lorenzo, L.; Ruibal-Lista, B.; Toro, S. (2020). Los primeros auxilios en el currículo de la educación obligatoria en España. Sportis Sci J, 6 (2), 365-389. DOI: https://doi.org/10.17979/sportis.2020.6.2.5831 


Scientific Technical Journal
Sportis. Revista Técnico-Cientifica del Deporte Escolar, Educación Física y Psicomotricidad
Sportis. Scientific Technical Journal of School Sport, Physical Education and Psychomotricity

CE: Criterios de evaluación; Comp: Competencias Clave.

Extraído de: RD 86/201.

\section{Discusión}

El análisis de la legislación curricular, muestra que los primeros auxilios han estado presentes en el currículo educativo, en mayor o menor medida, desde hace más de 40 años y con las últimas dos leyes de educación, LOE y LOMCE, ha ido ganando peso en el currículo.

Sin embargo, aunque el conocimiento de los ciudadanos españoles para afrontar una situación de emergencia y, en caso de ser necesario, proporcionar primeros auxilios ha aumentado en los últimos años (Gorjón, 2018), los últimos informes nos siguen situando a la cola de los países europeos (RACE, 2013; Ballesteros-Peña et al., 2015; SEMES, 2017). Un tercio de la población española no conoce cuál es el número único de emergencias europeo, y más de la mitad no ha recibido nunca formación específica en primeros auxilios y maniobras de soporte vital (Gorjón, 2018).

Esta falta de formación y seguridad de la población para actuar como primer interviniente, puede aplicarse a uno de los accidentes más comunes: parada cardíaca extrahospitalaria (Ballesteros-Peña et al. 2013; Berdowski et al., 2010; SEMES, 2017), en que la intervención precoz ante ella es fundamental para incrementar las probabilidades de supervivencia (Perales et al., 2010; Fothergill et al., 2013; Wissenberg et al., 2013). Por este motivo, el aprendizaje de maniobras de RCP y Soporte Vital Básico (SVB), se ha recomendado en todos los ámbitos de la sociedad, incluida la escuela (Lockey et al, 2016). Sin embargo, más del $60 \%$ de la población no sabría cómo reaccionar ante una parada cardiorrespiratoria y tan solo el 40,0 \% sería capaz de usar un desfibrilador externo automático (DEA) en caso de ser necesario (Gorjón, 2018).

Para citar este artículo utilice la siguiente referencia: Rodríguez-Lorenzo, L.; Ruibal-Lista, B.; Toro, S. (2020). Los primeros auxilios en el currículo de la educación obligatoria en España. Sportis Sci J, 6 (2), 365-389. DOI: https://doi.org/10.17979/sportis.2020.6.2.5831 


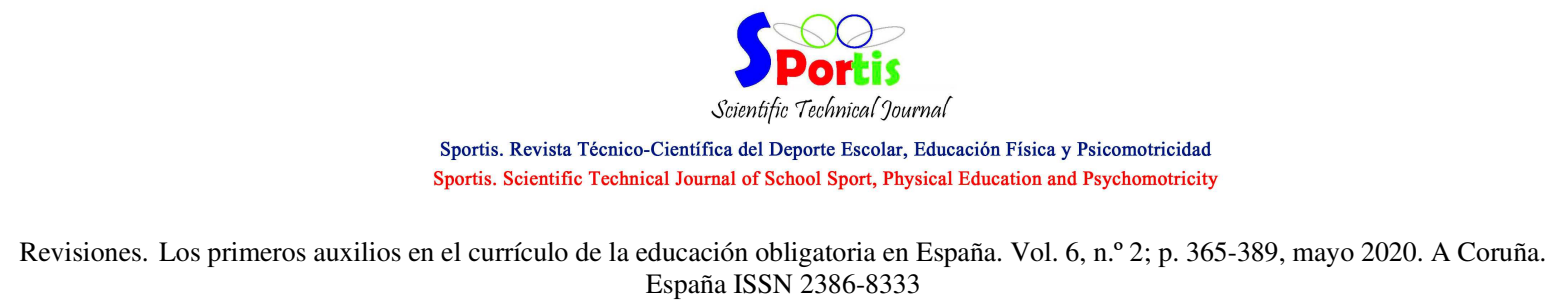

Tal y como se ha comentado, aunque la escuela haya sido designada como un lugar idóneo para la formación en primeros auxilios, algunos autores apuntan a que los docentes no suelen impartir estos contenidos debido a la falta de tiempo, recursos materiales y/o de la formación necesaria para llevarlos a cabo, y los que lo incluyen no parecen hacerlo de la forma más adecuada (Reder \& Quan, 2003; Rodríguez et al., 2014; Navarro-Patón et al., 2015; Navarro-Patón et al., 2016). La falta de formación puede provocar temor a impartir estos contenidos, aunque tiene fácil solución, ya que se ha demostrado que, tanto los futuros profesores de enseñanza secundaria (Ruibal-Lista et al., 2019) como los profesores de educación primaria y secundaria son capaces de impartir con éxito contenidos relacionados con los primeros auxilios tras un período breve de formación (Martín, 2015; Pichel-López et al., 2017), y que los propios docentes estarían dispuestos a impartirlos si se les forma previamente (López et al., 2008). Otros autores recomiendan que la formación de los docentes en primeros auxilios comience durante sus estudios universitarios (Navarro-Patón et al, 2017; Abelairas-Gómez et al, 2019).

Con respecto a la falta de tiempo y material, se ha comprobado que en la actualidad existen una amplia variedad de recursos didácticos (López-Izquierdo., 2019) y materiales (Lago-Ballesteros et al., 2018) para enseñar los primeros auxilios. Por otro lado, existen diferentes iniciativas o programas de enseñanza donde se conceden formación y material a los centros para poder impartir contenidos relacionados con los primeros auxilios en las aulas. Un ejemplo es el programa "RCP na aula", donde profesionales de los servicios de emergencias se encargan de formar a los profesores de educación física, como proveedores de RCP y uso del DEA (López et al., 2012), para que estos formen, a su vez a otros profesores y alumnos. Otra iniciativa, que presenta resultados positivos a la hora de formar a los profesores de los centros educativos es el "programa Anxos" (Pichel-López, 2017), impulsada por un grupo de profesores de la universidad de Vigo, para formar a escolares de educación infantil y primaria. O los talleres que imparte la Sociedad Española de Medicina de Urgencias y Emergencias en centros educativos, para aprender a identificar los síntomas y los pasos a seguir ante una parada cardíaca (Gorjón, 2018).

Por su parte, el alumnado de educación primaria y secundaria ha demostrado ser capaz aprender de forma adecuada maniobras de primeros auxilios, mejorando de forma 
significativa aspectos como: la llamada al número de emergencias, el reconocimiento de signos vitales, la colocación de accidentados en la posición adecuada y las técnicas de reanimación (Miró et al., 2008; Fleischhackl et al, 2009; López et al., 2012; Brown et al., 2018).
\end{abstract}

\title{
5. Conclusiones
}

La presencia de los primeros auxilios como contenido curricular ha ido variando en la legislación educativa desde 1970 hasta la actualidad. A partir del 2006, los primeros auxilios han ganado peso en el currículo, respaldado por la importancia de realizar una primera intervención ante un accidente, especialmente en la ESO y en la asignatura de Educación Física.

Numerosos estudios han demostrado que el alumnado de educación primaria y secundaria puede aprender maniobras de primeros auxilios de forma eficaz y que los profesores pueden ejercer de instructores si reciben formación previamente. Sin embargo, los primeros auxilios siguen sin ser impartidos por un gran número de profesores. Por todo esto, consideramos necesario de un Plan Nacional de formación y sensibilización en primeros auxilios en los centros escolares.

\section{Referencias bibliográficas}

Abelairas-Gómez, C., Carballo-Fazanes, A., Martínez-Isasi, S., López-García, S., Rico- Díaz, J., \& Rodríguez-Nuñez, A. (2019). Conocimiento y actitudes sobre los primeros auxilios y soporte vital básico de docentes de Educación Infantil y Primaria y los progenitores, Anales de Pediatría, In press. DOI: https://doi.org/10.1016/j.anpedi.2019.10.010

Abernethy, L., \& MacAuley, D. (2003). Impact of school sports injury. Britain Journal of Sports Medidcine. 37: 354-356. DOI: https://doi.org/10.1136/bjsm.37.4.354

Abraldes, J. A. (2007). Salvamento acuático. Guía del alumno. Murcia, España: Quaderna Editorial. 
Ballesteros-Peña S, Fernández-Aedo I, Pérez-Urdiales I, García-Azpiazu Z, Unanue-Arza S. (2016). Conocimientos y actitudes de los ciudadanos del País Vasco sobre la resucitación cardiopulmonar y los desfibriladores externos automatizados. $\begin{array}{llll}\text { Medicina } & \text { Intensiva. } & \text { 70(2): }\end{array}$ https://doi.org/10.1016/j.medin.2015.10.004
\end{abstract}

Ballesteros-Peña, S., Abecia-Inchaurregui, L. C., \& Echevarría-Orella, E. (2013). Factores asociados a la mortalidad extrahospitalaria de las paradascardiorrespiratorias atendidas por unidades de soporte vital básico en el País Vasco. Revista Española de Cardiología, 66(4), 269-274. DOI: ～https://doi.org/10.1016/j.recesp.2012.09.016

Berdowski, J., Berg, R. A., Tijssen, J. G., \& Koster, R. W. (2010). Global incidences of outof-hospital cardiac arrest and survival rates: Systematic review of 67 prospective studies. $\begin{array}{llll}\text { Resuscitation. } & 81: & 1479-1487 . & \text { DOI: }\end{array}$ https://doi.org/10.1016/j.resuscitation.2010.08.006

Brown, L. E., \& Dillon, W. C. (2018). CPR skill retention in 795 high school students following a 45-minute course with psychomotor practice. American Journal of $\begin{array}{llll}\text { Emergency } \quad \text { Medicine, } & \text { 36(6), }\end{array}$ https://doi.org/10.1016/j.ajem.2017.10.026

DADO. (2011). Programa de prevención de Lesiones: Detección de accidentes domésticos y de ocio. Instituto Nacional de Consumo. Recuperado de http://www.aecosan.msssi.gob.es/AECOSAN/docs/documentos/consumo/estudios/inform e_DADO_2011_2012.pdf

Decreto 105/2014, do 4 de setembro, polo que se establece o currículo da educación primaria na Comunidade Autónoma de Galicia

Decreto 86/2015, do 25 de xuño, polo que se establece o currículo da educación secundaria obrigatoria e do bacharelato na Comunidade Autónoma de Galicia. 


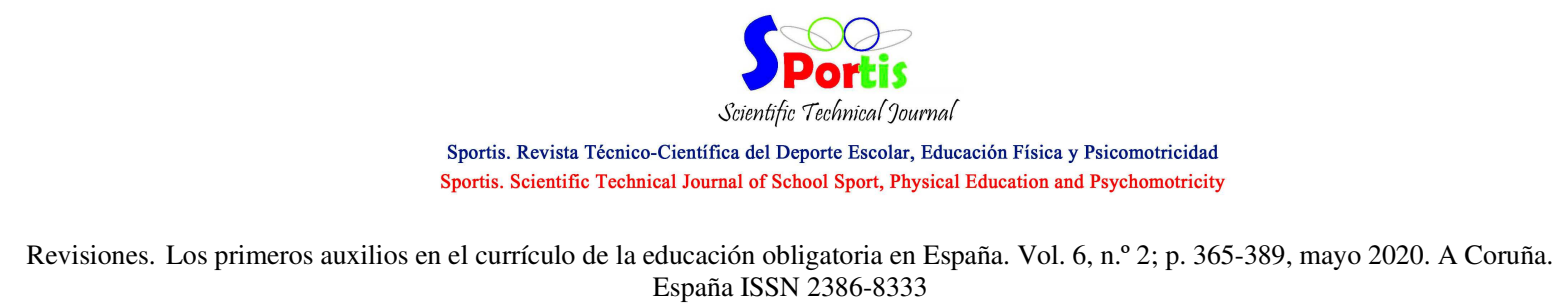

Fleischhackl, R., Nuernberger, A., Sterz, F., et al. (2009). School children sufficiently apply life supporting first aid: a prospective investigation. Critical Care. 13(4):R127. DOI: https://doi.org/10.1186/cc7984

Flórez, J. M., Romero-Martín, M. R., \& Izco, M. C. (2015). La educación física en el sistema educativo español. Revista Española de Educación Física y Deportes. 411 : 35-51. DOI: https://doi.org/10.17345/ute.2016.2.1006

Fothergill, R. T., Watson, L. R., Chamberlain, D., Virdi, G. K., Moore, F. P., \& Whitbread, M. (2013). Increases in survival from out-of-hospital cardiac arrest: a five year study. Resuscitation. 84: 1089-1092. DOI: https://doi.org/10.1016/j.resuscitation.2013.03.034

Gorjón, M. (2018). Conocimiento de la sociedad española en maniobras básicas de soporte vital y actitud ante las emergencias. Sociedad Española de Medicina de Urgencias y Emergencias. Recuperado de: https://noticias.fundacionmapfre.org/wpcontent/uploads/2018/10/Informe-Conocimiento-de-la-sociedad-espan\%CC\%83ola-enmaniobras-ba\%CC\%81 sicas-de-soporte-vital-final.pdf

IFRC. (2009). First Aid for a Safer Future: Focus on Europe. International Federation of Red Cross and Red Crescent Societies: Health and care department. Recuperado de https://www.ifrc.org/PageFiles/53459/First\%20aid\%20for\%20a\%20safer\%20future\%20F ocus\%20on\%20Europe \%20\%20Advocacy\%20report\%202009.pdf?epslanguage=en

Lago-Ballesteros, J., Basanta-Camiño, S., \& Navarro-Patón, R. (2018). La enseñanza de los primeros auxilios en educación física: revisión sistemática acerca de los materiales para su implementación. Retos, 39, 349-355.

Ley 14/1970, de 4 de agosto, General de Educación y Financiamiento de la Reforma Educativa.

Ley Orgánica 1/1990, de 3 de octubre, de ordenación general del sistema educativo. Boletín Oficial del Estado. 


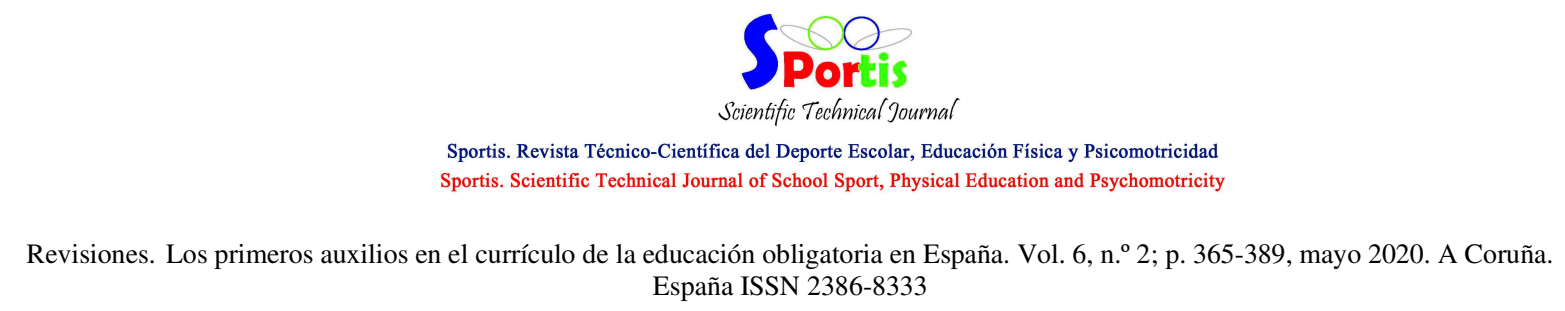

Ley Orgánica 10/2002, de 23 de diciembre, de Calidad de la Educación.

Ley Orgánica 2/2006, de 3 de mayo, de Educación.

Ley Orgánica 8/2013, de 9 de diciembre, para la mejora de la calidad educativa.

Lockey, A. S., Barton, K., \& Yoxall, H. (2016). Opportunities and barriers to cardiopulmonary resuscitation training in English secondary schools. European Journal of Emergency Medicine, 23, 381-385. DOI: https://doi.org/10.1097/MEJ.0000000000000307

López-Izquierdo, J. (2019). Propuesta de un proyecto interdisciplinar para el fomento del aprendizaje de los primeros auxilios en educación física. Emásf, Revista Digital de Educación Física, 60, 91-105.

López M., Garrote, A., Freire, M., Pérez, E., Rodríguez, A., \& Mosquera,M. (2008). Encuesta a profesores de Institutos de Secundaria sobre la enseñanza de la reanimación cardiopulmonar básica en sus centros. Emergencias. 20: 251-255.

López, M., Freire, M., Pérez, E., Chayán, M., Rasines, R., \& Iglesias, A. (2012). RCP na aula: programa de enseñanza de la reanimación cardiopulmonar básica para estudiantes de secundaria. Emergencias, 24(1):74-80.

Martín, R. A. (2015). Educación para la salud en primeros auxilios dirigida al personal docente del ámbito escolar. Enfermería universitaria, 12(2), 88-92. DOI: https://doi.org/10.1016/j.reu.2015.04.004

MESS. (2017). Anuario de Estadísticas sociolaborales. Ministerio de Empleo y Seguridad Social. Recuperado de http://www.mitramiss.gob.es/es/estadisticas/anuarios/2017/ATR/ATR.pdf

Miró, O., Escalada, X., Jiménez-Fábrega, X., Díaz, N., Sanclemente, G., Gómez, X., et al. (2008). Programa de Reanimación Cardiopulmonar Orientado a Centros de Enseñanza Secundaria (PROCES): conclusiones tras 5 años de experiencia. Emergencias. 20(4): 229236. DOI: https://doi.org/10.1157/13070434 


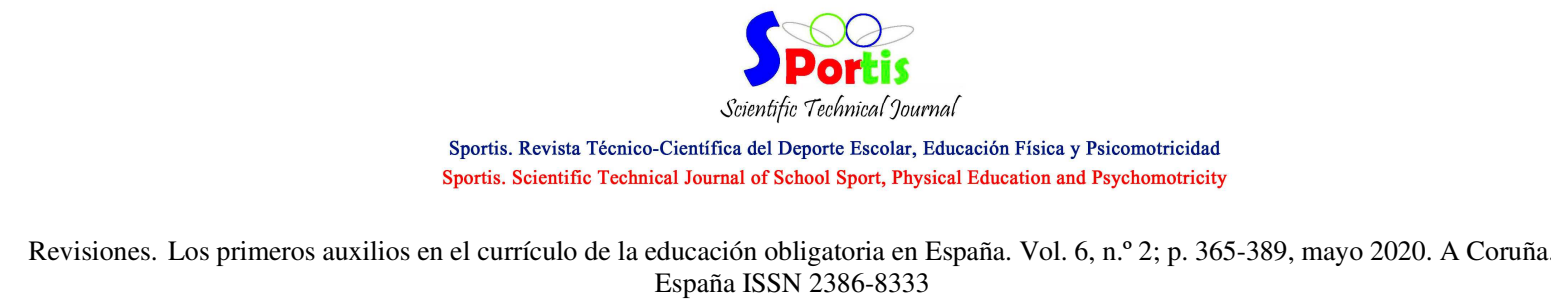

Molina, P., Valenciano-Valcárcel, J., \& Úbeda-Colomer, J. (2016). El diseño curricular de la Educación Física en España: Una revisión crítica desde la LOGSE a la LOMCE. Cultura, Ciencia y Deporte. 11(32): 97-106. DOI: https://doi.org/10.12800/ccd.v11i32.710

Navarro-Patón, N., Teijeiro, G. P., \& Camiño, S. B. (2016). ¿Tienen las futuras maestras y maestros de educación primaria la formación necesaria para iniciar las maniobras de reanimación cardiopulmonar en caso de emergencia escolar? Un estudio descriptivo. Educar. 52(1): 149-168.

Navarro-Patón, R., Arufe-Giráldez, V., \& Camiño, S. B. (2015). Estudio descriptivo de la enseñanza de los primeros auxilios desde el profesorado de Educación Física en centros de educación primaria. Sportis. Scientific Journal of School Sport, $\quad$ Physical Education and Psychomotricity. 1(1): 35-52. DOI: https://doi.org/10.17979/sportis.2015.1.1.1400

Navarro-Patón, R., Freire-Tellado, M., Pavón-Prieto, M. P., Vázquez-López, D., Neira Pájaro, M., \& Lorenzana-Bargueiras, S. (2017). Dispatcher assisted Cardiopulmonary Resuscitation (CPR): Is it still important to continue teaching lay bystander CPR? Resuscitation. 35(4): 569-573. DOI: https://doi.org/10.1016/j.ajem.2016.12.014

Orden de 17 de enero de 1981 por la que se regulan las enseñanzas de Educación Preescolar y del Ciclo Inicial de la Educación General Básica.

Orden de 18 de septiembre de 1987 por la que se modifica parcialmente el anexo I de la de 22 de marzo de 1975, que aprobaba los programas del Bachillerato Unificado y Polivalente.

Orden de 22 de marzo de 1975 por la que se desarrolla el Decreto 160/1975, de 23 de enero, que aprueba el Plan de Estudios del Bachillerato, y se regula el Curso de Orientación Universitaria.

Orden de 25 de noviembre de 1982 por la que se regulan las enseñanzas del Ciclo Superior de la Educación General Básica. 


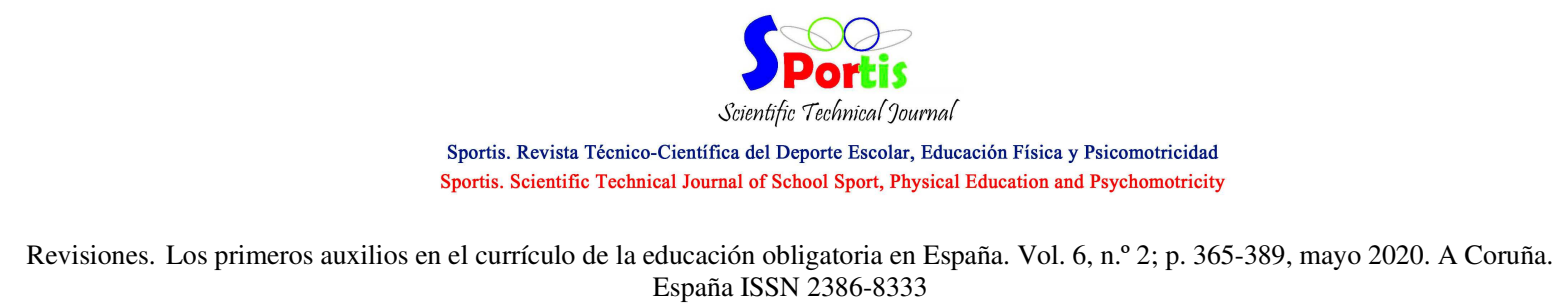

Orden de 6 de mayo de 1982, por la que se regulan las enseñanzas del Ciclo Medio de la Educación General Básica.

Peiró-Pérez, R., Seguí-Gómez, M., Pérez-González, C., Miralles-Espí, M., López Maside, A., \& Benavides, F. G. (2006). Lesiones por tráfico, de ocio y domésticas y laborales. Descripción de la situación en España. Gaceta Sanitaria, (20): 32-40. DOI: https://doi.org/10.1157/13086024

Perales N, Pérez JL, Pérez C. (2010). Respuesta comunitaria a la muerte súbita: resucitación cardiopulmonar con desfibrilación temprana. Revista Española de Cardiología Suplementos, 10, 21-31. DOI: https://doi.org/10.1016/S1131-3587(10)701084

Pichel-López, M., Martinez-Isasi, S., Barcala-Furelos, R., Fernández-Méndez, F., Vázquez Santamarina, D., Sánchez-Santos, L., et al (2017). Un primer paso en la enseñanza del soporte vital básico en las escuelas: la formación de losprofesores. Anales de pediatría, 89(5): 265-271. DOI: https://doi.org/10.1016/j.anpedi.2017.11.002

RACE. (2013). EuroTest: Informe sobre primeros auxilios. Real Automóvil Club de España. Recuperado de: https://www.race.es/wp-content/uploads/2013/02/EUROTEST-SOBREPRIMEROS-AUXILIOS-2013.pdf

RAE. (2014). Diccionario de la lengua española. Madrid, España: Espasa

Real Decreto 1006/1991, de 14 de junio, por el que se establecen las enseñanzas mínimas correspondientes a la Educación Primaria.

Real Decreto 1178/1992, de 2 de octubre, por el que se establecen las enseñanzas mínimas del Bachillerato.

Real Decreto 126/2014, de 28 de febrero, por el que se establece el currículo básico de la Educación Primaria.

Real Decreto 1345/1991, de 6 de septiembre, por el que se establece el currículo de la Educación Secundaria Obligatoria.

Para citar este artículo utilice la siguiente referencia: Rodríguez-Lorenzo, L.; Ruibal-Lista, B.; Toro, S. (2020). Los primeros auxilios en el currículo de la educación obligatoria en España. Sportis Sci J, 6 (2), 365-389. DOI: https://doi.org/10.17979/sportis.2020.6.2.5831 


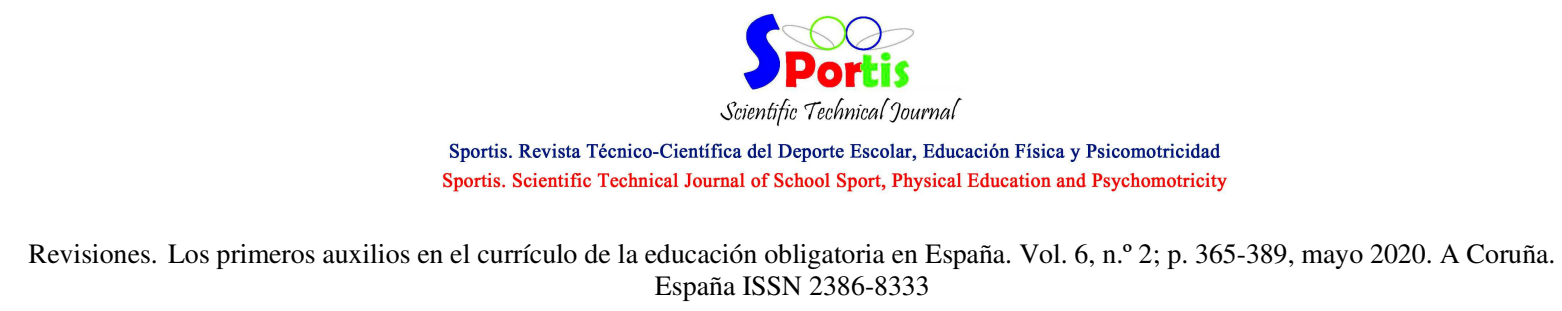

Real Decreto 1467/2007, de 2 de noviembre, por el que se establece la estructura del bachillerato y se fijan sus enseñanzas mínimas.

Real Decreto 1513/2006, de 7 de diciembre, por el que se establecen las enseñanzas mínimas de la Educación primaria.

Real Decreto 1631/2006, de 29 de diciembre, por el que se establecen las enseñanzas mínimas correspondientes a la Educación Secundaria Obligatoria.

Real Decreto 3087/1982, de 12 de noviembre, por el que se fijan las enseñanzas mínimas para el ciclo superior de Educación General Básica.

Real Decreto 3473/2000, de 29 de diciembre, por el que se modifica el Real Decreto 1007/1991, de 14 de junio, por el que se establecen las enseñanzas mínimas correspondientes a la educación secundaria obligatoria.

Real Decreto 69/1981, de 9 de enero, de ordenación de la Educación General Básica y fijación de las enseñanzas mínimas para el Ciclo Inicial.

Real Decreto 710/1982, de 12 de febrero, por el que se fijan las enseñanzas mínimas para el ciclo medio de la Educación General Básica.

Real Decreto 1105/2014, de 26 de diciembre, por el que se establece el currículo básico de la Educación Secundaria Obligatoria y del Bachillerato.

Reder, S. \& Quan, L. (2003). Cardiopulmonary resuscitation training in Washington state public high schools. Resuscitation. 56(3): 283-288. DOI: https://doi.org/10.1016/S0300-9572(02)00376-3

Rodríguez, M., Landázuri, M., Roaf, P., \& Beltrán, C. (2014). La actitud de los profesores de educación primaria ante los primeros auxilios. Revista de Educación y Desarrollo, 1(1): 20.

Ruibal-Lista, B., Aranda-García, S., López-García, S., Prieto, J. A., Castillo-Obeso del, M., \& Palacios-Aguilar, J. (2019). Effect of 45-minute CPR Training on Future Physical Para citar este artículo utilice la siguiente referencia: Rodríguez-Lorenzo, L.; Ruibal-Lista, B.; Toro, S. (2020). Los primeros auxilios en el currículo de la educación obligatoria en España. Sportis Sci J, 6 (2), 365-389. DOI: https://doi.org/10.17979/sportis.2020.6.2.5831 
Revisiones. Los primeros auxilios en el currículo de la educación obligatoria en España. Vol. 6, n. ${ }^{\text {2 }}$; p. 365-389, mayo 2020. A Coruña. España ISSN 2386-8333

Education Teachers. Apunts. Educación Física y Deportes, 138, 1-10. DOI: https://doi.org/10.5672/apunts.2014-0983.es.(2019/4).138.05

Ruíz-Fernández, Z. y Abraldes, JA. (2016). Determinación de variables identificativas sobre el socorrista acuático en dibujos de escolares de primaria. FEGUI. Revista de Salvamento Acuático y Primeros Auxilios, 43(5), 25-34.

Tenorio, D., Escobar, J. M., Stid Garzón, E., Castaño, C., \& Acevedo, A. (2009).Efectividad de intervenciones educativas en primeros auxilios. Investigaciones Andina, 18(11): 11-94.

Wilks, J., \& Pendergast, D. (2017). Skills for life: First aid and cardiopulmonary resuscitation in schools. Health Education Journal. 76(8): 1009-1023. DOI: https://doi.org/10.1177/0017896917728096

Wissenberg, M., Lippert, F. K., Folke, F., Weeke, P., Hansen, C. M., Christensen, E. F., et al. (2013). Association of national initiatives to improve cardiac arrest management with rates of bystander intervention and patient survival after out-of-hospital cardiac arrest. Journal of the American Medical Association. 310: 1377-1384. DOI: https://doi.org/10.1001/jama.2013.278483 